\title{
The role of the orbitofrontal cortex and the nucleus accumbens for craving in alcohol use disorder
}

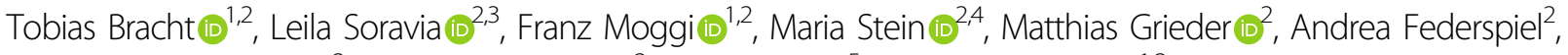
Raphaela Tschümperlin², Hallie M. Batschelet ${ }^{2}$, Roland Wiest ${ }^{5}$ and Niklaus Denier ${ }^{1,2}$

\begin{abstract}
This study aimed to investigate structural and functional alterations of the reward system and the neurobiology of craving in alcohol use disorder (AUD). We hypothesized reduced volume of the nucleus accumbens (NAcc), reduced structural connectivity of the segment of the supero-lateral medial forebrain bundle connecting the orbitofrontal cortex (OFC) with the NAcc (OFC-NAcc), and reduced resting-state OFC-NAcc functional connectivity (FC).

Furthermore, we hypothesized that craving is related to an increase of OFC-NAcc FC. Thirty-nine recently abstinent patients with AUD and 18 healthy controls (HC) underwent structural (T1w-MP2RAGE, diffusion-weighted imaging (DWI)) and functional (resting-state fMRI) MRI-scans. Gray matter volume of the NAcc, white matter microstructure (fractional anisotropy (FA)) and macrostructure (tract length) of the OFC-NAcc connection and OFC-NAcc FC were compared between AUD and HC using a mixed model MANCOVA controlling for age and gender. Craving was assessed using the thoughts subscale of the obsessive-compulsive drinking scale (OCDS) scale and was correlated with OFC-NAcc FC. There was a significant main effect of group. Results were driven by a volume reduction of bilateral NAcc, reduced FA in the left hemisphere, and reduced tract length of bilateral OFC-NAcc connections in AUD patients. OFC-NAcc FC did not differ between groups. Craving was associated with increased bilateral OFC-NAcc FC. In conclusion, reduced volume of the NAcc and reduced FA and tract length of the OFC-NAcc network suggest structural alterations of the reward network in AUD. Increased OFC-NAcc FC is associated with craving in AUD, and may contribute to situational alcohol-seeking behavior in AUD.
\end{abstract}

\section{Introduction}

Severe alcohol use disorder (AUD) is a chronic relapsing disorder associated with harmful somatic, psychological, and social consequences ${ }^{1}$. Core features of AUD are craving, the recurring desire and urge for alcohol consumption and the impaired ability to control alcoholseeking behavior despite its negative consequences ${ }^{2,3}$. According to the World Health Organization (WHO) more than 3 million deaths worldwide are caused by harmful use of alcohol each year ${ }^{4}$. AUD is characterized

Correspondence: Tobias Bracht (tobias.bracht@upd.unibe.ch)

${ }^{1}$ University Hospital of Psychiatry and Psychotherapy, University of Bern, Bern, Switzerland

${ }^{2}$ Translational Research Center, University Hospital of Psychiatry and Psychotherapy, University of Bern, Bern, Switzerland

Full list of author information is available at the end of the article by very high rates of post-residential treatment relapse $(80-92 \%)^{5,6}$, especially in the first 3 months after residential treatment ${ }^{7,8}$. Thus, there is a need for more effective treatment regimens. A profound knowledge on the neurobiology of addiction, particularly regarding craving and inhibition of alcohol seeking behavior, may contribute to improving novel treatment approaches such as alcohol-specific inhibition training, neurofeedback, or brain stimulation therapies ${ }^{9-11}$. This, in turn may set the path for a more personalized AUD treatment.

On a neurobiological level the reward system plays an essential role for the pathophysiology of addiction ${ }^{2}$, in particular regarding the hedonic experience of consuming alcohol (e.g., liking) and the urge for alcohol consumption (e.g., wanting) ${ }^{12}$. Central relay stations of the reward system are the ventral tegmental area (VTA), the nucleus

\section{(c) The Author(s) 2021}

(c) (i) Open Access This article is licensed under a Creative Commons Attribution 4.0 International License, which permits use, sharing, adaptation, distribution and reproduction cc) in any medium or format, as long as you give appropriate credit to the original author(s) and the source, provide a link to the Creative Commons license, and indicate if changes were made. The images or other third party material in this article are included in the article's Creative Commons license, unless indicated otherwise in a credit line to the material. If material is not included in the article's Creative Commons license and your intended use is not permitted by statutory regulation or exceeds the permitted use, you will need to obtain permission directly from the copyright holder. To view a copy of this license, visit http://creativecommons.org/licenses/by/4.0/. 
accumbens (NAcc), and the prefrontal cortex (PFC) including the orbitofrontal cortex (OFC), a core region for the selection of motivationally relevant events ${ }^{2,3,13}$. In healthy participants including heavy social drinkers, liking is moderately associated with wanting, although it has been suggested that wanting and liking are dividable and independent processes ${ }^{14}$. During the progression of addiction there is a decrease of liking and an increase of wanting, the latter being related to the experience of craving and to compulsive alcohol use ${ }^{15}$. Wanting relies on dopaminergic pathways projecting from the VTA to the NAcc and to the OFC ${ }^{12}$. These dopaminergic projections initiate neuroplastic changes in their target regions (e.g., the OFC), which encode for learned associations with pleasurable events (e.g., alcohol consumption $)^{16}$. Such associations may form the basis for situational alcohol-seeking behavior in AUD. Consequently, models of addiction refer to the OFC-NAcc connection as the final common pathway for initiating alcohol-seeking behavior in AUD ${ }^{2,3}$.

Gray matter volume reductions of core regions of the reward system in AUD have been reported repeatedly (e.g., NAcc, OFC ${ }^{17,18}$ ). This may be due to a particularly pronounced vulnerability of regions of the reward system to the neurotoxic effects of alcohol ${ }^{19,20}$. Structurally, those regions of the reward system are connected through white matter pathways forming a complex network ${ }^{13}$. The most commonly applied measure for the assessment of white matter microstructure is the fractional anisotropy (FA), reflecting the coherence of diffusion of water molecules ${ }^{21}$. A series of voxel-by-voxel comparison-based whole brain studies found FA reductions in AUD ${ }^{22,23}$. Localizations of findings are widespread (e.g., in the corpus callosum, internal and external capsule, or in the fornix) and many findings lack associations with behavioral measures relevant to addiction. Thus, those alterations may rather be the result of alcohol toxicity than a neurobiological correlate of addiction pathophysiology. This assumption is also supported by observations that global white matter microstructure alterations in AUD are reversible, if abstinence is maintained and recur after relapse $\mathrm{e}^{24}$.

The fiber architecture of the reward system ${ }^{13}$, believed to underlie the pathophysiology of addiction ${ }^{2,3}$, is complex because of multiple crossing and merging fibers ${ }^{25}$. In such regions of crossing fibers, tract-specific conclusions require the use of tractography, allowing for a noninvasive in vivo reconstruction of neuronal pathways based on diffusion weighted MRI ${ }^{26,27}$. However, tractography studies of the reward system in AUD are scarce $^{28,29}$. One previous study used tractography to investigate the supero-lateral branch of the medial forebrain bundle (slMFB) in $\mathrm{AUD}^{28}$. The slMFB consists of mono- and polysynaptic projection pathways of the VTA $\left(\operatorname{vtaPP}^{25,30}\right.$,) connecting the VTA with the NAcc and with prefrontal brain regions including the $\mathrm{OFC}^{31}$. Based on lesion studies in humans and on tract-tracing studies, such projection pathways likely consist of bidirectional corticopetal and corticofugal projection pathways into and out of the $\mathrm{VTA}^{25,32}$. In our study, we investigate the sIMFB segment anterior of the NAcc, which predominantly connects to the OFC $\mathrm{OC}^{30,33,34}$. In the following, we refer to this segment of the sIMFB as the OFC-NAcc connection. In addition to FA, a measure of white matter microstructure ${ }^{27}$, we use the tract length as a measure of white matter macrostructure, which may be related to white matter atrophy ${ }^{35}$ complementing our structural white matter analyses.

Functional MRI (fMRI) allows for the measurement of fluctuations in blood oxygen level-dependent (BOLD) signals that are associated with specific brain functions. This complements structural analyses of gray matter volume and white matter microstructure. In AUD, there are decreased BOLD-levels of core regions of the reward system in the absence of alcoholic stimuli ${ }^{3,18,22,36-38}$. Extending classical BOLD analyses, resting-state functional connectivity (FC) analyses provide crucial information on connectivity strengths between spatially segregated brain regions, hereby yielding functional networks $^{39}$. Decreased resting-state FC between core regions of the reward system including the NAcc and the $\mathrm{OFC}^{37,38,40}$ may be associated with a lack of response to natural rewarding stimuli and result in a psychological state characterized by feelings of anxiety, reduced energy, and restlessness ${ }^{2,41}$.

Consistent with the assumption that patients with severe AUD drink alcohol to diminish aversive affects ${ }^{42}$, there is an enhanced cue reactivity in AUD in response to alcoholic cues in regions of the reward system ${ }^{18,22,36,43,44}$. The OFC and the NAcc seem to be key to the neurobiology of AUD given that both regions have been related to craving in response to alcoholic cues ${ }^{44-46}$. In this study, we extend these previous fMRI analyses linking craving at rest to resting-state OFC-NAcc connectivity, hereby providing information on the neurobiology of craving on a functional network level.

It was the aim of this first three-fold multimodal MRIstudy using gray matter volumetry, tractography, and FC analyses to investigate structural and functional aspects of the OFC-NAcc network, and its association with craving in a group of recently abstinent patients with severe AUD compared to healthy controls. Our first hypothesis was decreased bilateral volume of the NAcc in AUD $^{17,18}$. Second, we hypothesized decreased FA and a reduced tract length of the OFC-NAcc connection in AUD ${ }^{22}$. Third, we hypothesized decreased OFC-NAcc restingstate FC in $\mathrm{AUD}^{37,38,40}$ and fourth, we hypothesized that increased resting-state OFC-NAcc FC is associated with craving in AUD ${ }^{44,46}$. 


\section{Methods}

\section{Study participants}

All AUD-patients were participating in a clinical trial investigating the effects of an alcohol-inhibition training on relapse $\mathrm{e}^{47}$. A sub-sample of those AUD-patients was included in a longitudinal fMRI-study investigating the functional correlates of this training. The diffusionweighted MRI-protocol is an extension of the latter study and was added at a later stage. In our study, only baseline MRI-scans and assessments that were acquired before the intervention started were considered. To avoid influences of age-related atrophy on brain structure inclusion criterion for all participants was age between 18-59 years of age. Thirty-nine right-handed detoxified patients with severe AUD attending a 12-week abstinence-oriented residential treatment program for AUD in a specialized treatment center in Switzerland (Clinic Suedhang) were recruited. All patients met criteria for AUD as clinically assessed in accordance with the DSM-5, and had been abstinent for at least 4 weeks prior to study participation. Eighteen right-handed healthy controls (HC) with non-problematic drinking behavior (Alcohol Use Disorders Identification Test $\left(\mathrm{AUDIT}^{48}\right)$ score $<8$; Alcohol Use Disorder Scale (AUD-S ${ }^{49}$ score $\left.<2\right)$ ), and low scores regarding psychopathology (Brief Symptom Check List $\left(\mathrm{BSCL}^{50}, \mathrm{GSI}_{t \text {-value }} \leq 63\right)$ were recruited. Current treatment for a psychiatric diagnosis and/or psychopharmacological medication, treatment for any substance use disorder in the past, problematic substance use (except nicotine; Drug Use Disorders Identification Test (DUDIT) score $\leq 8$ per substance ${ }^{51}$, e.g., cannabis) and neurocognitive problems were exclusion criteria.

This study aimed to investigate the neurobiology of subjective craving at rest (as assessed with resting-state fMRI). Craving was assessed immediately before the MRIscan and measured with the German version of the obsessive compulsive drinking scale (OCDS $)^{52}$. The OCDS consists of 14 items assessing both obsessive thoughts about alcohol use and compulsive drinking behavior. Given that resting-state fMRI is measured in the absence of behavior by definition, we chose the obsessive thoughts of alcohol subscale as our outcome measure for craving. Furthermore, we used the Comprehensive Alcohol Expectancy Questionnaire (CAEQ), a self-report measure with 41 items, designed to assess an individual's positive and negative alcohol outcome expectancies, which are related to the level of alcohol use and relapse following treatment for alcohol use disorders ${ }^{53}$. In addition, the brief symptom checklist (global severity index, GSI) was used for the assessment of subjective impairments due to somatic and mental problems ${ }^{54}$. Depressive symptoms were assessed with the Beck depression inventory $(\mathrm{BDI})^{55}$. All participants provided written informed consent and were reimbursed for participation. The study was approved by the local ethics committee (KEK-number: 2016-00988) and registered at Clinicaltrials.gov (NCT02968537) and the Swiss National Clinical Trials Portal (SNCTP000002043).

\section{MRI-data acquisition}

MRI data were acquired at the University Hospital of Bern, with a 3 Tesla Siemens Magnetom Prisma scanner and with a 64-channel head/neck coil. For high-resolution T1-weighted structural images, we used a bias-field corrected MP2RAGE sequence for improved segmentation (256 Slices, field of view $(\mathrm{FOV})=256 \times 256,256 \times 256$ matrix, $1 \mathrm{~mm}^{3}$ isotropic resolution, repletion time $(\mathrm{TR})=$ $5000 \mathrm{~ms}$, echo time $(\mathrm{TE})=2.98 \mathrm{~ms}$, inversion time $T 1=$ $700 \mathrm{~ms}$ and $T 2=2500 \mathrm{~ms}$ ). The MP2RAGE sequence generated three image volumes, two gradient echo images (INV1 and INV2) and a T1 weighted image (UNI). Diffusion weighted images (DWI) were acquired using a spin-echo echo-planar sequence with 64 non-collinear directions and $b$-value $=1000 \mathrm{~s} / \mathrm{mm}^{2}$ (60 Slices, FOV $=$ $269 \times 269,128 \times 128$ matrix, $2.2 \mathrm{~mm}^{3}$ isotropic resolution, $\mathrm{TR}=6200 \mathrm{~ms}, \mathrm{TE}=69 \mathrm{~ms})$. A resting-state fMRI scan (condition: eyes closed) was acquired using whole brain echo planar imaging (EPI) (300 volumes with 60 slices per volume, FOV $=230 \times 230,104 \times 104$ matrix, $2.2 \mathrm{~mm}^{3}$ isotropic resolution, $\mathrm{TR}=1300 \mathrm{~ms}, \mathrm{TE}=37 \mathrm{~ms}$ ).

\section{Data analyses \\ Structural MRI pre-processing}

We used FSL-FIRST, a model-based segmentation and registration tool for subcortical structures implemented in FSL 5.0 (http://www.fmrib.ox.ac.uk/fsl/first/index.html), for segmentation of bilateral NAcc ${ }^{56,57}$. Due to noisy background of the MP2RAGE UNI image, we used the INV2 image for brain extraction and binary mask generation with FSL-BET using robust brain center estimation (-R option). Then, we applied the binary mask to the UNI image using FSLMATHS and run FSL-FIRST for segmentation of the bilateral NAcc using the brain extracted UNI image as input and standard settings. The segmentation model uses a Bayesian appearance model and measures the probabilistic relationships between shape and GM intensity. Subcortical volumes (in $\mathrm{mm}^{3}$ ) of the bilateral NAcc were finally determined using FSLSTATS with the all_fast_firstseg files as input and a threshold of 25.5-26.5 for the left NAcc and 57.5-58.5 for the right NAcc (Fig. 1A).

\section{Diffusion-weighted MRI data pre-processing}

Diffusion-weighted MRI (DW-MRI) data were analyzed using ExploreDTI 4.8.6 ${ }^{58}$. Data were pre-processed as described in previous publications ${ }^{59,60}$. In short, a subjection motion and distortion correction and an EPIcorrection was performed ${ }^{61}$. Whole-brain tractography 


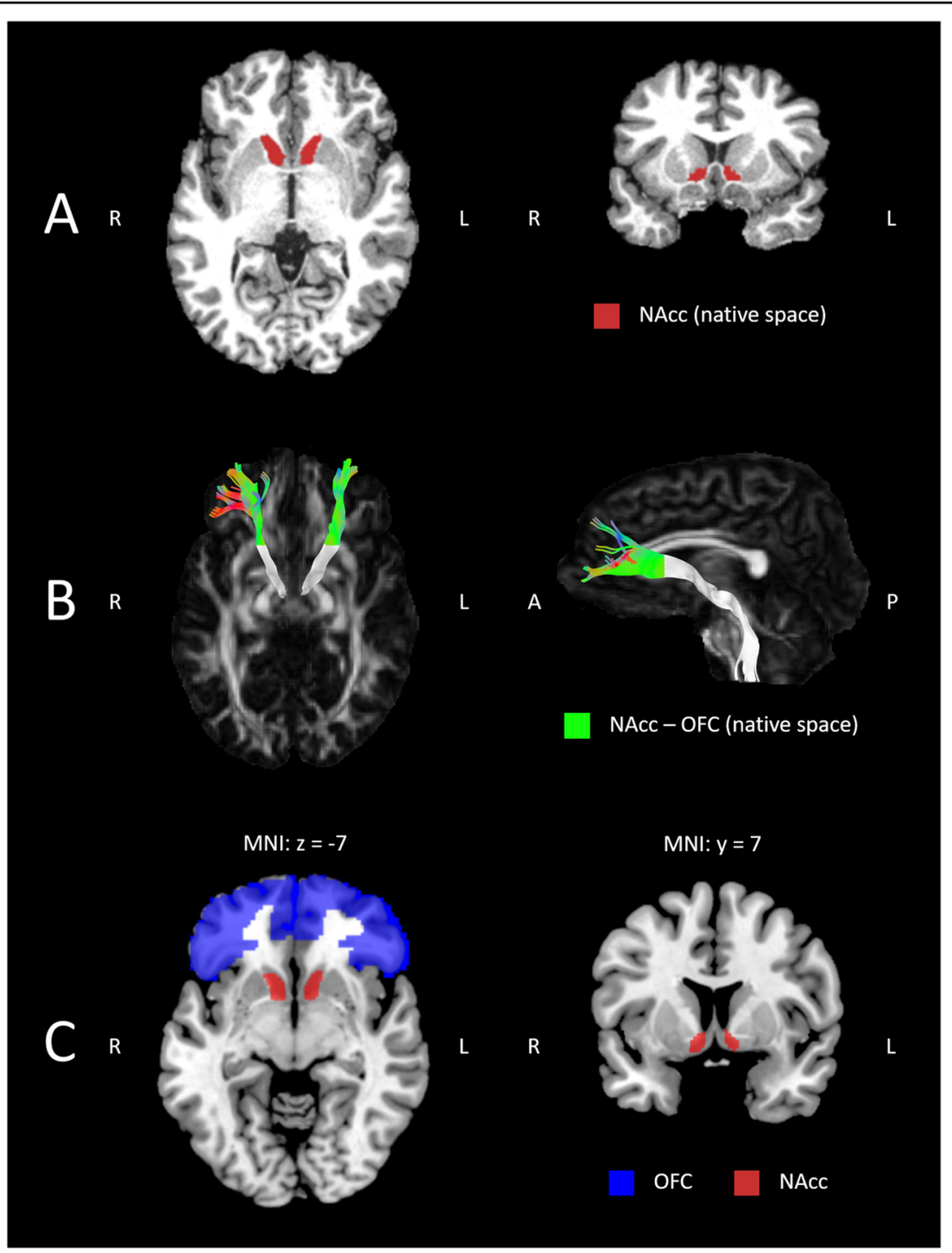

Fig. 1 Multimodal imaging analyses. A Example volumetry of bilateral NAcc in native space. B Example of individual native space OFC-NAcc connections (color coded) and the remaining proximal segment (in gray). C OFC (BA 10 and 11) and NAcc masks in MNI space for computation of bilateral OFC-NAcc FC.

was executed fitting a single diffusion tensor model to the DW-MRI data ${ }^{62}$. Angle threshold of $>45^{\circ}$ and FA $<0.2$ were used as termination criteria. Diffusion properties (e.g., FA) were sampled along the tracts.

\section{Tract reconstruction}

We aimed at reconstructing the OFC-NAcc segment of the sIMFB, because this segment is part of a polysynaptic network connecting OFC, NAcc and $\mathrm{VTA}^{31}$, core regions for the pathophysiology of craving in $\mathrm{AUD}^{2,3}$. First, the entire slMFB was reconstructed as described in previous publications $^{34,35}$. The following seed gates were used separately for both hemispheres: The VTA was identified on a horizontal section. A circular seed was drawn anterior of the red nucleus, medial of the substantia nigra and posterior to the mammillary bodies. A second seed region was drawn on a coronal section at the height of the NAcc surrounding the anterior limb of the internal capsule. Seed regions were chosen in line with previous studies (e.g., ${ }^{34,35}$ ). Given that we were specifically interested in the OFC-NAcc segment of the MFB, we used the splitter tool implemented in ExploreDTI 4.8.6 to isolate the slMFB segment anterior of the NAcc connecting to (predominantly orbitofrontal) prefrontal brain regions (see Fig. 1B). Mean-FA and tract length were calculated for each tract $^{35}$. 


\section{Functional MRI pre-processing}

We analysed FC using a seed-driven approach of the CONN 19c toolbox ${ }^{63}$. We used standard pipeline and parameters. Pre-processing steps included realignment and field map correction of EPI volumes, co-registration to structural volumes (MP2RAGE) and segmentation/ normalization of structural volumes and smoothing of normalized EPI volumes. We applied a band-pass filtering $(0.008-0.09 \mathrm{~Hz})$ to remove physiological signals and regressed nuisance variables including 12 realignment parameters and each five time series within segmented white matter and cerebrospinal fluid, derived by principal component analysis. Afterwards, we defined the left and right NAcc as seed region and computed their regional FC over the whole brain. Using MATLAB, we extracted mean values of FC-maps within the left and right OFC using predefined masks representing Brodmann area 10 and 11 of the WFUPickatlas 3.0 (see Fig. 1C).

\section{Statistical analyses}

Based previous studies investigating functional ${ }^{37}$ and structural alterations ${ }^{17,28}$ of the reward system in AUD, we expected to get a mean effect size of $f^{2}=0.15$ for the between factor (AUD vs. HC) having a total sample size of 57 participants and performing a mixed-model MANCOVA with one between factor, one within factor, four dependent variables and controlling for the two covariates age and gender. A posthoc analysis to calculate the power achieved, given alpha $=0.05$, sample size $=57$, number of groups $=2$, number of dependent variables $=4$, and number of covariates $=3$, yielded a power (1-beta) of 0.93 using the program $\mathrm{G}^{*}$ Power $^{64}$.

Statistical analyses were performed using Statistical Package for Social Sciences SPSS 26.0 (SPSS, Inc., Chicago, Illinois). Demographics between AUD und HC were compared using $t$-tests for continuous variables or $\chi^{2}$ tests for dichotomous variables. A mixed-model MANCOVA controlling for age and gender with the between-factor group ( $\mathrm{HC}$ vs. AUD), the within-factor hemisphere (left, right) and four dependent variables comprising the four modalities (gray matter volume of NAcc, FA (OFC-NAcc), tract length (OFC-NAcc) and FC (OFC-NAcc)) was used. Significant main effects were followed up with post-hoc tests. In case of a significant group $\times$ hemisphere interaction, post-hoc ANCOVAs controlling for age and gender were calculated separately for each hemisphere and modality. All tests were two-tailed and a probability of $<0.05$ was considered statistically significant. Effect sizes were reported as $\eta^{2,65}$. Additional exploratory correlations for the AUD group were calculated between the four imaging modalities and the total OCDS-scale and the compulsive drinking subscale (see Supplementary material Tables 1 and 2). To provide more detailed anatomical information on the localization of FC, two separate seed-based FC analyses were performed using the NAcc as a seed. First, NAcc-FC to the whole brain and second to the OFC mask. Analyses were performed separately for each hemisphere using the CONN toolbox ${ }^{63}$. Voxel-wise comparisons in FC between AUD and HC groups were performed with a voxel-level threshold of $p<$ 0.001 and with a family wise error (FWE) cluster-level correction of $p<0.05$.

\section{Results \\ Study population}

The groups did not differ regarding age or gender. Results indicate that our AUD group mainly consists of patients with severe AUD (see Table 1 for demographics and clinical characteristics).

\section{Group comparisons}

The mixed model MANCOVA revealed a significant main effect of group showing a large effect size $(F(4,50)=$ 2.612, $p=0.046, \eta^{2}=0.173$ ). Posthoc tests demonstrated a significant group main effect for NAcc volume (AUD patients < controls; $F(1,53)=5.986, p=0.018, \eta^{2}=$ 0.101 , hemisphere $\times$ group interaction $(F(1,53)=0.458$, $\left.p=0.502, \eta^{2}=0.009\right)$. Further posthoc tests revealed a significant hemisphere $\times$ group interaction for FA

Table 1 Sociodemographic and clinical characteristics.

\begin{tabular}{llll}
\hline & $\begin{array}{l}\text { AUD patients } \\
(\boldsymbol{N}=\mathbf{3 9})\end{array}$ & $\begin{array}{l}\text { Healthy controls } \\
(\boldsymbol{N}=\mathbf{1 8})\end{array}$ & $\boldsymbol{p}$-values \\
\hline Females/males & $15 / 24$ & $6 / 12$ & 0.709 \\
Age & $41.92(8.55)$ & $40.44(12.12)$ & 0.645 \\
Relationship (yes/no) & $20 / 19$ & $15 / 3$ & $0.021^{*}$ \\
Years of education & $14.21(3.76)$ & $16.33(3.46)$ & $0.047^{*}$ \\
Employment (yes/no) & $23 / 16$ & $17 / 1$ & $0.007^{* *}$ \\
Number of & $2.89(2.92)$ & & \\
detoxifications & & & \\
Days of abstinence & $30.32(14.65)$ & & $<0.001^{* * *}$ \\
Years of problematic & $11.25(9.17)$ & & $<0.001^{* * *}$ \\
drinking & & & $<0.001^{* * *}$ \\
AUDIT & $24.43(7.25)$ & $4.00(1.88)$ & \\
AUD & $29.40(6.83)$ & $0.83(1.30)$ & $<0.001^{* * *}$ \\
OCDS-Thoughts & $4.59(3.85)$ & $0.11(0.32)$ & \\
CAEG & $3.37(0.46)$ & & $0.18(0.19)$ \\
BDI II & $15.70(9.17)$ & $1.21(0.61)$ & \\
BSCL GSI & & & \\
\hline
\end{tabular}

Means and standard deviations are displayed. Group differences are displayed by ${ }^{*} p<0.05$, ** $p<0.01$, and ${ }^{* * *} p<0.001$.

AUDIT alcohol use disorders identification test, AUD alcohol use disorder scale, OCDS obsessive compulsive drinking scale, CAEG comprehensive alcohol expectancy questionnaire, $B D I$ Beck depression inventory, Brief symptom check list. 


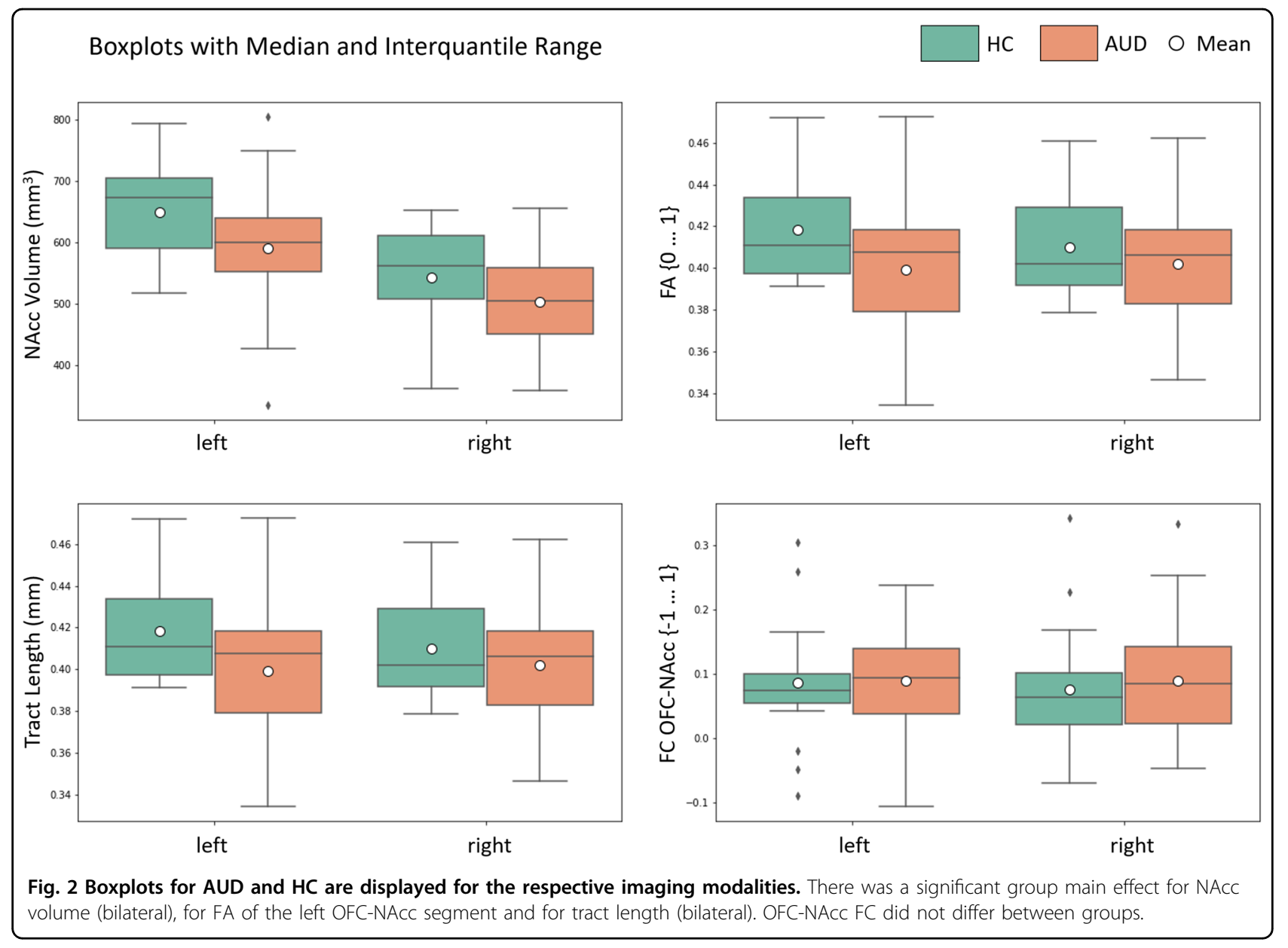

$\left(F(1,53)=4.629, p=0.036, \eta^{2}=0.080\right)$. Therefore, separate ANCOVAs with the independent variable group and the dependent variable FA controlling for age and gender were calculated for each hemisphere. FA of the OFC-NAcc connection was reduced in AUD in the left $\left(F(1,53)=5.224, p=0.026, \eta^{2}=0.090\right)$, but not in the right $\left(F(1,53)=0.803, p=0.374, \eta^{2}=0.015\right)$ hemisphere. Finally, there was a significant main effect for OFC-NAcc tract length (AUD patients < controls; $F(1,53)=4.609$, $p=0.036, \eta^{2}=0.080$, hemisphere $\times$ group interaction $\left(F(1,53)=0.015, p=0.902, \eta^{2}<0.001\right)$, but not for resting state OFC-NAcc FC $(F(1,53)=0.152, p=0.698$, $\eta^{2}=0.003$, hemisphere $\times$ group interaction $(F(1,53)=$ $0.198, p=0.659, \eta^{2}=0.004$ ) (see Fig. 2). For both hemispheres, we found no significant group differences between AUD and HC in seed-based FC from the NAcc to the whole brain and to the OFC mask. See supplementary Fig. 1 for visualization of whole-brain FC within group and hemisphere.

\section{Correlations with craving and OFC-NACC FC}

In the AUD group craving was significantly correlated with OFC-NAcc FC for both the left $(r=0.477, p=0.002)$ and the right ( $r=0.390, p=0.014)$ hemisphere, indicating higher FC with increased craving (see Fig. 3).

\section{Discussion}

This is the first study that combined gray matter volume analyses of the NAcc, tractography of the OFC-NAcc segment of the sIMFB and OFC-NAcc FC to investigate structural and functional alterations in AUD and its association with craving. Our results point to structural alterations of the OFC-NAcc network in recently abstinent patients suffering from severe AUD. We found reduced gray matter volume of the NAcc, and impaired structural connectivity in terms of reduced white matter microstructure (FA) of the left and macrostructure (tract length) of bilateral OFC-NAcc connections. There were no group differences in resting-state OFC-NAcc FC. Bilateral OFC-NAcc resting-state FC positively correlated with craving, thus underpinning this network's key role for individual variations in the pathophysiology of AUD.

One anatomical concept regarding the OFC-NAcc pathway is that this final common pathway activates the $\mathrm{NAcc}^{2}$, which in turn projects to the pallidum initiating alcohol-seeking behavior via thalamo-cortical pathways ${ }^{3}$. 

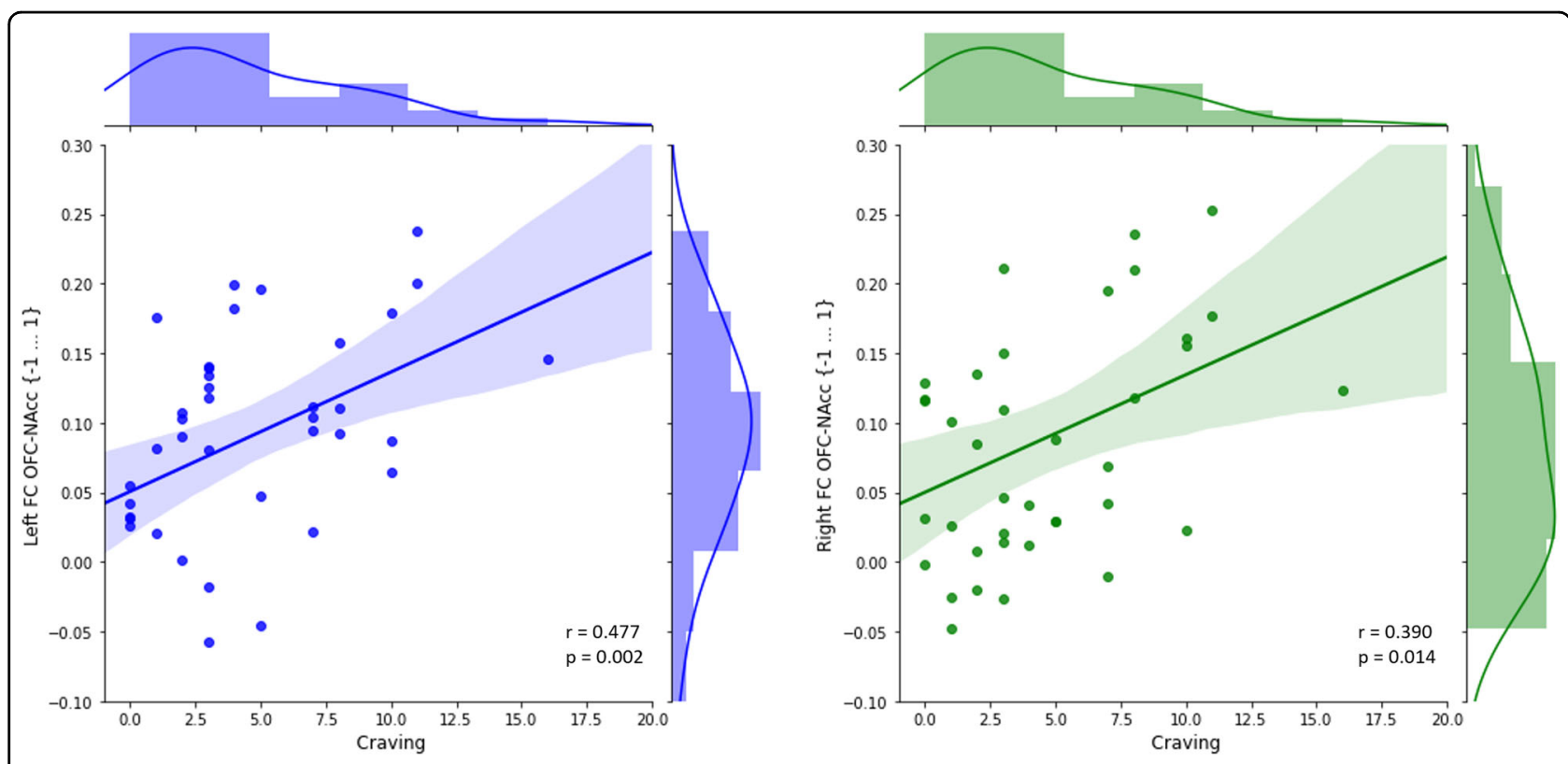

Fig. 3 Correlations of OFC-NACC FC with craving in AUD.

An alternative explanation is that OFC-NAcc projections of the slMFB activate corticofugal glutamatergic projections from the NAcc to the VTA (as visualized by the gray shaded segment of the slMFB (Fig. 1B)). Dopaminergic efferents from the VTA may project to the PFC via the infero-medial MFB (the classic anatomical MFB description in rodents $)^{33,66}$, hereby mediating craving. Both theories are consistent with results of exploratory correlations suggesting that higher FA of the left OFC-NAcc is associated with increased craving in AUD $(r=0.321, p=$ 0.046 , see supplementary material, Table 1$)$. Thus, white matter microstructure of the OFC-NAcc pathway may indeed be associated with craving, indicating a core role of this pathway for the pathophysiology of addiction.

This assumption is indirectly supported by our restingstate fMRI-results. We identified a positive correlation between left and right resting-state OFC-NAcc FC and craving. Consequently, the OFC-NAcc network may play an important role for craving, even at rest and in the absence of alcoholic stimuli. The intrusiveness of craving allows for an analogy with obsessive compulsive disorder (OCD). Interestingly, pathophysiology of OCD has also been related to increased structural connectivity between the OFC and the $\mathrm{NAcc}^{25}$. Our finding thus complements event-related fMRI studies demonstrating increased BOLD activations of the NAcc, and the OFC in response to alcoholic cues ${ }^{36,44}$. A previous event-related FC study using alcoholic cues investigated craving in a clinically heterogeneous patient group ranging from social drinkers to severe $\mathrm{AUD}^{45}$. Both positive (medial OFC-insula) and negative (lateral OFCNAcc) correlations between FC during a cue reactivity task and craving were identified ${ }^{45}$. Differences in study design (resting-state vs. cue reactivity task) and clinical differences could account for the inconsistencies. Furthermore, craving may either be driven by reward (e.g., in social drinkers ${ }^{67}$ ) or by avoidance of negative affect (e.g., in severe $\mathrm{AUD}^{42}$ ). Thus, it is difficult to compare the neurobiology of craving between clinically diverse AUD-groups.

In line with our hypothesis, we found reduced volume of bilateral NAcc in AUD, thus replicating previous studies (e.g., ${ }^{68,69}$ ). Furthermore, reduced FA of the left and reduced tract length of bilateral OFC-NAcc connections were found in AUD. Exploratory analysis showed an association $(r=0.387, p=0.015$, see Supplementary material Table 2) between the left NAcc volume and FA of the left OFC-NAcc segment. Thus, one may speculate whether alcohol-induced gray matter atrophy of the NAcc leads to reduced white matter microstructure in AUD. Given that the directionality (in terms of afferents or efferents) of fiber tracts cannot be determined based on tractography, it may also be possible that white matter microstructure alterations stem from cortical atrophy in the OFC, a common and well replicated finding in $\mathrm{AUD}^{70}$. Another explanation is that the toxic effects of alcohol damage both gray and white matter of the OFC-NAcc network $^{19,20,71}$ or that white matter damage of the OFCNAcc connection leads to retrograde atrophy of NAcc gray matter $^{72}$. In either case, three complementary structural measures (FA and tract length of the OFCNAcc connection and NAcc-volume) suggest atrophy of the OFC-NAcc network in AUD, most likely a consequence of alcohol-induced neurotoxicity ${ }^{19,20,71}$. 
Contrary to our hypothesis, we did not find any group differences in resting-state OFC-NAcc FC between AUD and healthy controls. Previous studies demonstrated lower resting-state $\mathrm{FC}$ of the reward system in abstinent AUD patients than in $\mathrm{HC}^{37}$, a finding that is more pronounced in long-term than in short term AUD abstinence $^{38}$. Given that our AUD group mainly consists of short-term abstinent patients this may have contributed to the absence of group differences regarding resting-state OFC-NAcc FC. However, previous findings are not entirely consistent. One longitudinal study demonstrated that contrary to the initial hypothesis, relapsers had lower FC between the PFC and the NAcc than abstainers ${ }^{73}$. Overall, divergent findings may stem from comparing results of anatomically adjacent but functionally complementary brain regions (e.g., different hotspots for wanting and liking within the NAcc shell or functional differences regarding the medial and the lateral $\mathrm{OFC}^{74,75}$. Furthermore, it is crucial to note that FC analyses are influenced by a series of factors such as AUD severity and duration, the presence of comorbid depressive symptoms, the duration of abstinence or the contextual situation regarding alcohol exposure, which may as well partially explain heterogeneous findings ${ }^{38,42,45}$.

Finally, this study has some limitations: First, our correlational study does not allow for conclusions regarding causalities or directionalities. Second, we use tractography to reconstruct individual projection pathways. However, it is impossible to determine the anatomical directionality of projection pathways. Furthermore, it is impossible to make statements on direct and indirect connections and on the type of neurotransmitters (e.g., dopaminergic or glutamatergic). Third, although AUD patients and healthy controls are well matched and analysis are controlled for age and gender, there remain differences (e.g., depressive symptoms, years of education). Given that education is strongly correlated with the group variable using education as covariate can reduce validity or sensitivity and is not advised ${ }^{76}$. Thus, we cannot statistically rule out that education may have had an influence on neurophysiological results. Fourth, our sample of AUD patients comprises almost exclusively patients with severe AUD. While this is a positive aspect allowing for a precise definition of the sample under investigation, it limits generalizability to patients with mild or moderate AUD.

To conclude, our findings in recently abstinent patients with severe AUD demonstrate that AUD is associated with gray and white matter structural alterations of the OFC-NAcc network, putatively a neurotoxic effect of alcohol. Functionally, this network plays a core role for craving as suggested by the identified correlation between craving and resting-state OFC-NAcc FC, which may also affect white matter microstructure of the corresponding OFC-NAcc connection pathway. Future studies should assess if those changes reverse with abstinence in the long term $^{24}$. Furthermore, it is of clinical interest to assess the predictive value of such MRI-assessments for novel treatment approaches, such as neurofeedback, alcoholinhibition training, or brain stimulation therapies ${ }^{9-11}$.

\section{Acknowledgements}

The study was funded by a grant from the Swiss Foundation for Alcohol Research (SSA-Nr:: 283), by the Swiss National Science Foundation (SNF; No: 105319_159286) and by the Novartis-Foundation for Medical-Biological Research (No: 19A063). Tobias Bracht received a scholarship from the Adrian et Simone Frutiger Foundation. We thank Sara Lustenberger, Miranda Germann, Elena Leumann, Kirstin Schürch, Sophia Vögtli, Manuela Wüthrich, and Benjamin Erb for excellent research assistance. Special thanks goes to Dr. Nadja Razavi for initiating fruitful research collaborations.

\section{Author details}

'University Hospital of Psychiatry and Psychotherapy, University of Bern, Bern, Switzerland. ${ }^{2}$ Translational Research Center, University Hospital of Psychiatry and Psychotherapy, University of Bern, Bern, Switzerland. ${ }^{3}$ Clinic Suedhang, Kirchlindach, Switzerland. ${ }^{4}$ Department of Clinical Psychology and

Psychotherapy, Institute of Psychology, University of Bern, Bern, Switzerland. ${ }^{5}$ Institute of Diagnostic and Interventional Neuroradiology, University of Bern, Bern, Switzerland

\section{Conflict of interest}

The authors declare that the research was conducted in the absence of any commercial or financial relationships that could be construed as a potential conflict of interest.

\section{Publisher's note}

Springer Nature remains neutral with regard to jurisdictional claims in published maps and institutional affiliations.

Supplementary information The online version contains supplementary material available at https://doi.org/10.1038/s41398-021-01384-w.

Received: 18 December 2020 Revised: 12 April 2021 Accepted: 19 April 2021

Published online: 04 May 2021

\section{References}

1. Rehm, J. The risks associated with alcohol use and alcoholism. Alcohol Res. Health 34, 135-143 (2011).

2. Volkow, N. D., Koob, G. F. \& McLellan, A. T. Neurobiologic advances from the brain disease model of addiction. N. Engl. J. Med. 374, 363-371 (2016).

3. Kalivas, P. W. \& Volkow, N. D. The neural basis of addiction: a pathology of motivation and choice. Am. J. Psychiatry 162, 1403-1413 (2005).

4. WHO. Based on the Global Status Report on Alcohol and Health 2018. (WHO, 2018).

5. Heinz, A., Beck, A., Grusser, S. M., Grace, A. A. \& Wrase, J. Identifying the neural circuitry of alcohol craving and relapse vulnerability. Addict. Biol. 14, 108-118 (2009).

6. Brandon, T. H., Vidrine, J. I. \& Litvin, E. B. Relapse and relapse prevention. Annu. Rev. Clin. Psychol. 3, 257-284 (2007)

7. Harvey, R., Jason, L. A. \& Ferrari, J. R. Substance abuse relapse in Oxford House recovery homes: a survival analysis evaluation. Subst. Abus. 37, 281-285 (2016).

8. Stohs, M. E., Schneekloth, T. D., Geske, J. R., Biernacka, J. M. \& Karpyak, V. M. Alcohol craving predicts relapse after residential addiction treatment. Alcohol Alcohol. 54, 167-172 (2019).

9. Batschelet, H. M., Stein, M., Tschuemperlin, R. M., Soravia, L. M. \& Moggi, F. Alcohol-specific computerized interventions to alter cognitive biases: a systematic review of effects on experimental tasks, drinking behavior, and neuronal activation. Front. Psychiatry 10, 871 (2019).

10. Bari, A. et al. Neuromodulation for substance addiction in human subjects: a review. Neurosci. Biobehav. Rev. 95, 33-43 (2018). 
11. Dousset, C. et al. Preventing relapse in alcohol disorder with EEGneurofeedback as a neuromodulation technique: a review and new insights regarding its application. Addict. Behav. 106, 106391 (2020).

12. Berridge, K. C. \& Robinson, T. E. Liking, wanting, and the incentive-sensitization theory of addiction. Am. Psychol. 71, 670-679 (2016).

13. Haber, S. N. \& Knutson, B. The reward circuit: linking primate anatomy and human imaging. Neuropsychopharmacology 35, 4-26 (2010).

14. Hobbs, M., Remington, B. \& Glautier, S. Dissociation of wanting and liking for alcohol in humans: a test of the incentive-sensitisation theory. Psychopharmacology 178, 493-499 (2005).

15. Berridge, K. C., Robinson, T. E. \& Aldridge, J. W. Dissecting components of reward: 'liking', 'wanting', and learning. Curr. Opin. Pharm. 9, 65-73 (2009).

16. Jay, T. M. Dopamine: a potential substrate for synaptic plasticity and memory mechanisms. Prog. Neurobiol. 69, 375-390 (2003).

17. Shim, J. H., Kim, Y. T., Kim, S. \& Baek, H. M. Volumetric reductions of subcortical structures and their localizations in alcohol-dependent patients. Front. Neurol. 10, 247 (2019).

18. Buhler, M. \& Mann, K. Alcohol and the human brain: a systematic review of different neuroimaging methods. Alcohol Clin. Exp. Res. 35, 1771-1793 (2011).

19. Fernandes, Y., Rampersad, M. \& Gerlai, R. Embryonic alcohol exposure impairs the dopaminergic system and social behavioral responses in adult zebrafish. Int.J. Neuropsychopharmacology 18, 1-8 (2015).

20. Pascual, M., Boix, J., Felipo, V. \& Guerri, C. Repeated alcohol administration during adolescence causes changes in the mesolimbic dopaminergic and glutamatergic systems and promotes alcohol intake in the adult rat. J. Neurochem. 108, 920-931 (2009).

21. Basser, P. J. \& Pierpaoli, C. Microstructural and physiological features of tissues elucidated by quantitative-diffusion-tensor MRI. 1996. J. Magn. Reson. 213, 560-570 (2011).

22. Fritz, M., Klawonn, A. M. \& Zahr, N. M. Neuroimaging in alcohol use disorder: from mouse to man. J. Neurosci. Res. https://doi.org/10.1002/jnr.24423 (2019).

23. Fortier, C. B. et al. Widespread effects of alcohol on white matter microstructure. Alcohol Clin. Exp. Res. 38, 2925-2933 (2014).

24. Pfefferbaum, A. et al. White matter microstructural recovery with abstinence and decline with relapse in alcohol dependence interacts with normal ageing: a controlled longitudinal DTI study. Lancet Psychiatry 1, 202-212 (2014).

25. Coenen, V. A. et al. Tractographic description of major subcortical projection pathways passing the anterior limb of the internal capsule. Corticopetal organization of networks relevant for psychiatric disorders. Neuroimage Clin. 25, 102165 (2020).

26. Dell'Acqua, F. \& Tournier, J. D. Modelling white matter with spherical deconvolution: How and why? NMR Biomed. 32, e3945 (2019).

27. Jones, D. K., Knosche, T. R. \& Turner, R. White matter integrity, fiber count, and other fallacies: the do's and don'ts of diffusion MRI. Neuroimage 73, 239-254 (2013).

28. Rivas-Grajales, A. M. et al. Sexually dimorphic structural abnormalities in major connections of the medial forebrain bundle in alcoholism. Neuroimage Clin. 19, 98-105 (2018).

29. Morales, A. M., Jones, S. A. \& Harman, G., Patching-Bunch, J. \& Nagel, B. J. Associations between nucleus accumbens structural connectivity, brain function, and initiation of binge drinking. Addict. Biol. 25, e12767 (2019).

30. Coenen, V. A. et al. The anatomy of the human medial forebrain bundle: ventral tegmental area connections to reward-associated subcortical and frontal lobe regions. Neuroimage Clin. 18, 770-783 (2018).

31. Bracht, T. et al. White matter microstructure alterations of the medial forebrain bundle in melancholic depression. J. Affect. Disord. 155, 186-193 (2014).

32. Frankle, W. G., Laruelle, M. \& Haber, S. N. Prefrontal cortical projections to the midbrain in primates: evidence for a sparse connection. Neuropsychopharmacology 31, 1627-1636 (2006).

33. Coenen, V. A., Panksepp, J., Hurwitz, T. A., Urbach, H. \& Madler, B. Human medial forebrain bundle (MFB) and anterior thalamic radiation (ATR): imaging of two major subcortical pathways and the dynamic balance of opposite affects in understanding depression. J. Neuropsychiatry Clin. Neurosci. 24, 223-236 (2012).

34. Bracht, T., Doidge, A. N., Keedwell, P. A. \& Jones, D. K. Hedonic tone is associated with left supero-lateral medial forebrain bundle microstructure. Psychol. Med. 45, 865-874 (2015).

35. Denier, N. et al. Reduced tract length of the medial forebrain bundle and the anterior thalamic radiation in bipolar disorder with melancholic depression. J. Affect. Disord. 274, 8-14 (2020).
36. Schacht, J. P., Anton, R. F. \& Myrick, H. Functional neuroimaging studies of alcohol cue reactivity: a quantitative meta-analysis and systematic review. Addict. Biol. 18, 121-133 (2013).

37. Camchong, J., Stenger, A. \& Fein, G. Resting-state synchrony in long-term abstinent alcoholics. Alcohol Clin. Exp. Res. 37, 75-85 (2013).

38. Camchong, J., Stenger, V. A. \& Fein, G. Resting-state synchrony in short-term versus long-term abstinent alcoholics. Alcohol Clin. Exp. Res. 37, 794-803 (2013).

39. van den Heuvel, M. P. \& Hulshoff Pol, H. E. Exploring the brain network: a review on resting-state fMRI functional connectivity. Eur. Neuropsychopharmacol. 20, 519-534 (2010).

40. Muller-Oehring, E. M., Jung, Y. C., Pfefferbaum, A., Sullivan, E. V. \& Schulte, T. The resting brain of alcoholics. Cereb. Cortex 25, 4155-4168 (2015).

41. Hagele, C. et al. Dimensional psychiatry: reward dysfunction and depressive mood across psychiatric disorders. Psychopharmacology 232, 331-341 (2015).

42. Fein, G., Camchong, J., Cardenas, V. A. \& Stenger, A. Resting state synchrony in long-term abstinent alcoholics: effects of a current major depressive disorder diagnosis. Alcohol 59, 17-25 (2017).

43. Mitchell, J. M. et al. Alcohol consumption induces endogenous opioid release in the human orbitofrontal cortex and nucleus accumbens. Sci. Transl. Med. 4, 116 ra116 (2012).

44. Myrick, H. et al. Differential brain activity in alcoholics and social drinkers to alcohol cues: relationship to craving. Neuropsychopharmacology 29, 393-402 (2004).

45. Strosche, A. et al. Investigation of brain functional connectivity to assess cognitive control over cue-processing in alcohol use disorder. Addict. Biol. 26 , e12863 (2020).

46. Myrick, $\mathrm{H}$. et al. Effect of naltrexone and ondansetron on alcohol cue-induced activation of the ventral striatum in alcohol-dependent people. Arch. Gen. Psychiatry 65, 466-475 (2008).

47. Tschuemperlin, R. M., Stein, M., Batschelet, H. M., Moggi, F. \& Soravia, L. M. Learning to resist the urge: a double-blind, randomized controlled trial investigating alcohol-specific inhibition training in abstinent patients with alcohol use disorder. Trials 20, 402 (2019).

48. Babor, T. F., Higgins-Biddle, J. C., Saunders, J. B. \& Monteiro, M. G. Audit. The Alcohol Use Disorders Identification Test. Guidelines for Use in Primary Care (World Health Organization, 2001).

49. Wechsler, H., Davenport, A., Dowdall, G., Moeykens, B. \& Castillo, S. Health and behavioral consequences of binge drinking in college. A national survey of students at 140 campuses. JAMA 272, 1672-1677 (1994).

50. Franke G. H. Brief Symptom Inventory von L.R. Derogatis - Deutsches Manual. [Brief symptom Inventory of L.R. Derogatis - German Manual] (Göttingen, 2000).

51. Voluse, A. C. et al. Psychometric properties of the Drug Use Disorders Identification Test (DUDIT) with substance abusers in outpatient and residential treatment. Addict. Behav. 37, 36-41 (2012).

52. Anton, R. F., Moak, D. H. \& Latham, P. The obsessive compulsive drinking scale: a self-rated instrument for the quantification of thoughts about alcohol and drinking behavior. Alcohol Clin. Exp. Res. 19, 92-99 (1995).

53. Demmel, R. \& Hagen, J. The comprehensive alcohol excpectancy questionnaire: I. Scale development. Sucht 49, 292-299 (2003).

54. Franke, G. BSCL-Brief Symptom Checklist. (Hogrefe Verlag, 2017).

55. Beck, A. T., Ward, C. H., Mendelson, M., Mock, J. \& Erbaugh, J. An inventory for measuring depression. Arch. Gen. Psychiatry 4, 561-571 (1961).

56. Carlezon, W. A. Jr. \& Thomas, M. J. Biological substrates of reward and aversion: a nucleus accumbens activity hypothesis. Neuropharmacology 56, 122-132 (2009).

57. Patenaude, B., Smith, S. M., Kennedy, D. N. \& Jenkinson, M. A Bayesian model of shape and appearance for subcortical brain segmentation. Neuroimage $\mathbf{5 6}$, 907-922 (2011).

58. Leemans, A., Jeurissen, B., Sijbers, J. \& Jones, D. K. ExporeDTI: a graphical toolbox for processing, analyzing and visualizing diffusion MR data. Proceedings of the International Society for Magnetic Resonance in Medicine 17th Annual Meeting. Honolulu, Hawaii, 3536 (2009).

59. Bracht, T. et al. Physical activity is associated with left corticospinal tract microstructure in bipolar depression. Neuroimage Clin. 20, 939-945 (2018).

60. Bracht, T. et al. Increased structural connectivity of the medial forebrain bundle in schizophrenia spectrum disorders is associated with delusions of paranoid threat and grandiosity. Neuroimage Clin. 24, 102044 (2019).

61. Leemans, A. \& Jones, D. K. The B-matrix must be rotated when correcting for subject motion in DTI data. Magn. Reson. Med. 61, 1336-1349 (2009). 
62. Basser, P. J., Mattiello, J. \& LeBihan, D. Estimation of the effective self-diffusion tensor from the NMR spin echo. J. Magn. Reson. B 103, 247-254 (1994).

63. Whitfield-Gabrieli, S. \& Nieto-Castanon, A. Conn: a functional connectivity toolbox for correlated and anticorrelated brain networks. Brain Connect 2 125-141 (2012).

64. Faul, F., Erdfelder, E., Lang, A. G. \& Buchner, A. G*Power 3: a flexible statistical power analysis program for the social, behavioral, and biomedical sciences. Behav. Res. Methods 39, 175-191 (2007).

65. Olejnik, S. \& Algina, J. Generalized eta and omega squared statistics: measures of effect size for some common research designs. Psychol. Methods 8, 434-447 (2003).

66. MacNiven, K. H., Leong, J. K. \& Knutson, B. Medial forebrain bundle structure is linked to human impulsivity. Sci. Adv. 6, eaba4788 (2020).

67. Dager, A. D. et al. Functional magnetic resonance imaging (fMRI) response to alcohol pictures predicts subsequent transition to heavy drinking in college students. Addiction 109, 585-595 (2014).

68. Makris, N. et al. Decreased volume of the brain reward system in alcoholism. Biol. Psychiatry 64, 192-202 (2008).

69. Sullivan, E. V., Deshmukh, A., De Rosa, E., Rosenbloom, M. J. \& Pfefferbaum, A. Striatal and forebrain nuclei volumes: contribution to motor function and working memory deficits in alcoholism. Biol. Psychiatry 57, 768-776 (2005)

70. Tomasi, D. G. et al. Association between reduced brain glucose metabolism and cortical thickness in alcoholics: evidence of neurotoxicity. Int. J. Neuropsychopharmacol. 22, 548-559 (2019).

71. de la Monte, S. M. \& Kril, J. J. Human alcohol-related neuropathology. Acta Neuropathol. 127, 71-90 (2014).

72. Hurwitz, T. A., Mandat, T., Forster, B. \& Honey, C. Tract identification by novel MRI signal changes following stereotactic anterior capsulotomy. Stereotact. Funct. Neurosurg. 84, 228-235 (2006).

73. Camchong, J., Stenger, A. \& Fein, G. Resting-state synchrony during early alcohol abstinence can predict subsequent relapse. Cereb. Cortex 23 2086-2099 (2013).

74. Berridge, K. C. \& Kringelbach, M. L. Affective neuroscience of pleasure: reward in humans and animals. Psychopharmacology 199, 457-480 (2008).

75. Kringelbach, M. L. \& Rolls, E. T. The functional neuroanatomy of the human orbitofrontal cortex: evidence from neuroimaging and neuropsychology. Prog. Neurobiol. 72, 341-372 (2004).

76. Miller, G. A. \& Chapman, J. P. Misunderstanding analysis of covariance. J. Abnorm. Psychol. 110, 40-48 (2001). 